\title{
Systems biology research at the SBB young scientists school series
}

\author{
Y.L. Orlov ${ }^{1,2 *}$, L. Wong ${ }^{3}$, T.V. Tatarinova ${ }^{4}$, R. Hofestädt ${ }^{5}$, N.A. Kolchanov ${ }^{1}$ \\ ${ }^{1}$ Institute of Cytology and Genetics SB RAS, Novosibirsk, Russia \\ ${ }^{2}$ Novosibirsk State University, Novosibirsk, Russia \\ ${ }^{3}$ National University of Singapore (NUS), Singapore \\ ${ }^{4}$ University of La Verne, USA \\ ${ }^{5}$ Bielefeld University, Bielefeld, Germany \\ *e-mail:orlov@bionet.nsc.ru
}

Key words: education, bioinformatics, international conferences, BGRS conference series

The Institute of Cytology and Genetics of the Siberian Branch of the Russian Academy of Sciences (ICG SB RAS) hosts the International Multi-conference on Bioinformatics of Genome Regulation and StructurelSystems Biology (BGRS $\backslash S B$ ) every two years, beginning from 1998. From BGRS $\backslash$ SB'2008 onwards, the Young Scientists School on Systems Biology and Bioinformatics (SBB) runs as a satellite event following the BGRS $\backslash S B$ conference or as a standalone annual event. Since the first meeting, the SBB has grown into a large international event. Gradually, the initial focus has been extended from systems biology and classical bioinformatics topics to gene network analysis and reconstruction, and omics technologies [2].

The Journal of Bioinformatics and Computational Biology (JBCB) publishes special issues on bioinformatics, algorithms, network analysis dedicated to BGRS $\backslash \mathrm{SB}$. The first JBCB special issue in 2006 highlighted BGRS $\backslash$ SB-2006 [3]. Then JBCB published special issues on the 2012, 2014, and 2016 conferences [4-6]. Additionally, the journal publishes reports from the SBB schools. For instance, JBCB has published proceedings of SBB-2015 on modeling of gene network based on material presented at earlier BGRS $\backslash$ SB meetings $[3,5,6]$.

The Institute of Cytology and Genetics SB RAS organized in 2017 the Belyaev Conference-2017 on genetics and evolution, dedicated to the 100th anniversary of Academician, Professor Dmitry K. Belyaev (1917-1985), an outstanding scientist, evolutionist and geneticist [7]. The works reported at Belyaev Conference-2017 and SBB'2017 School on Computational Biology were recently covered in the special issues of several international journals: BMC Evolutionary Biology [7], BMC Genetics [8], BMC Plant Biology, BMC Genomics, BMC Neuroscience, Vavilov Journal of Selection and Breeding (http://vavilov.elpub.ru/jour/issue/view/32/showToc). Meanwhile, a recent JBCB special issue presents materials from SBB'2017 School on Systems Biology and Bioinformatics (SBB'2017), organized in June 2017 in Yalta, Russia [1].

Integration of bioinformatics data is central point of the meetings and Schools organized in Novosibirsk. Journal of Integrative bioinformatics (https:/www.degruyter.com/ view $/ \mathrm{j} / \mathrm{jib}$ ) collects papers on Integration of Data, Methods and Tools, Big Data [9].

Overall, we continue the organization of special journal issues collected works on bioinformatics and biomedical applications presented by young scientists, thus extending traditions of post-conference special journal issues of the BGRS $\backslash S B$ conference series. This year's series will highlight materials presented at BGRS $\backslash S B-2018$ multiconference; special issues are planned at several BMC journals, and Frontiers in Genetics, and JBCB. The JBCB special issue on BGRS-2018 is currently open for submission at the journal 
web-site; it is our pleasure to invite authors to submit materials to the JBCB BGRS-2018 special issue.

Acknowledgements: We shall acknowledge all the international reviewers who helped make JBCB special issues better, improve the science and presentation of the materials of the authors. The organization of education round table at BGRS-2018 multiconference has been supported by Russian Ministry of Science project 28.12487.2018/12.1.

\section{References}

1. Orlov Y.L., Tatarinova T.V., Zakhartsev M.V., Kolchanov N.A. (2018) Introduction to the 9th Young Scientists School on Systems Biology and Bioinformatics (SBB'2017). Journal Bioinformatics Computational Biology. 16(1):1802001 (5 pages). DOI 10.1142/S0219720018020018.

2. Baranova A.V., Orlov Y.L. (2016) The papers presented at 7th Young Scientists School "Systems Biology and Bioinformatics" (SBB'15): Introductory Note. BMC Genetics. 17(Suppl 1):20.

3. Kolchanov N.A., Hofestädt R., Orlov Y.L. (2007) Introduction to the selected papers of BGRS'06. Journal Bioinformatics Computational Biology. 5(2b):vii-viii.

4. Kolchanov N.A., Orlov Y.L., (2013) Introductory note for BGRS-2012 special issue. Journal Bioinformatics Computational Biology. 11(1):1302001.

5. Orlov Y.L., Hofestädt R., Kolchanov N.A. (2015) Introductory note for BGRS $\backslash$ SB-2014 special issue. Journal of Bioinformatics and Computational Biology. 13(1):1502001.

6. Orlov Y.L., Kolchanov N.A., Hofestädt R., Wong L. (2017) Editorial - Bioinformatics development at the BGRSISB conference series: 10th anniversary. Journal Bioinformatics Computational Biology. 15(2): 1702001.

7. Orlov Y.L., Baranova A.V., Herbeck Y.E. (2017) Evolutionary Biology at Belyaev Conference-2017. BMC Evol Biol. 17(Suppl 2):260.

8. Orlov Y.L., Baranova A.V., Tatarinova T.V., Kolchanov N.A. (2017) Genetics at Belyaev Conference-2017: introductory note. BMC Genetics. 18(Suppl 1):116.

9. Chen M., Harrison A., Shanahan H., Orlov Y. (2017) Biological Big Bytes: Integrative Analysis of Large Biological Datasets. J Integr Bioinform. 14(3). pii: /j/jib.2017.14.issue-3/jib-2017-0052/jib-2017-0052. $\mathrm{xml}$. 